\title{
AN AUTOMATIC QUADRATURE FOR CAUCHY PRINCIPAL VALUE INTEGRALS
}

\author{
TAKEMITSU HASEGAWA AND TATSUO TORII
}

\begin{abstract}
An automatic quadrature is presented for computing Cauchy principal value integrals $Q(f ; c)=f_{a}^{b} f(t) /(t-c) d t, a<c<b$, for smooth functions $f(t)$. After subtracting out the singularity, we approximate the function $f(t)$ by a sum of Chebyshev polynomials whose coefficients are computed using the FFT. The evaluations of $Q(f ; c)$ for a set of values of $c$ in $(a, b)$ are efficiently accomplished with the same number of function evaluations. Numerical examples are also given.
\end{abstract}

\section{INTRODUCTION}

We present an automatic quadrature scheme for approximating principal value integrals

$$
Q(f ; c)=f_{-1}^{1} \frac{f(t)}{t-c} d t, \quad-1<c<1,
$$

where $f(t)$ are assumed to be smooth functions. Piessens et al. [17] give an automatic quadrature program for evaluating $Q(f ; c)$ in $(1.1)$ for a single value of $c$.

In this paper, for a set of values of $c$ in $(-1,1)$ we efficiently compute a set of approximations $\left\{Q_{N}(f ; c)\right\}$ to the integrals $(1.1)$ satisfying the prescribed tolerance $\varepsilon_{a}$. To this end, it is required to construct quadrature rules which have error estimates independent of the values of $c$ for smooth functions $f(t)$.

Our method is an extension of the Clenshaw-Curtis method [4] (henceforth abbreviated to CC method) for the integral $\int_{-1}^{1} f(t) d t$ to the problem (1.1) [1], [2], [3], [14]. In the CC method, the function $f(t)$ is approximated by a sum of Chebyshev polynomials $T_{k}(t)$,

$$
p_{N}(t)=\sum_{k=0}^{N}{ }^{\prime \prime} a_{k}^{N} T_{k}(t), \quad-1 \leq t \leq 1,
$$

Received October 30, 1989.

1980 Mathematics Subject Classification (1985 Revision). Primary 65D30, 65D32; Secondary $41 \mathrm{A55}$.

Key words and phrases. Cauchy principal value integral, automatic integration, Chebyshev interpolation. 
interpolating $f(t)$ at the abscissae $t_{j}^{N}=\cos \left(\pi_{j} / N\right) \quad(0 \leq j \leq N)$, which are the zeros of the polynomial $\omega_{N+1}(t)$ defined by

$$
\omega_{N+1}(t)=T_{N+1}(t)-T_{N-1}(t)=2\left(t^{2}-1\right) U_{N-1}(t), \quad N \geq 1,
$$

where $U_{k}(t)$ is the Chebyshev polynomial of the second kind defined by $U_{k}(t)=$ $\sin (k+1) \theta / \sin \theta \quad(t=\cos \theta)$. In (1.2), the double prime denotes the summation where the first and last terms are halved. The truncated Chebyshev series (1.2) converges rapidly as $N$ increases if $f(t)$ is a smooth function.

Chawla and Kumar [3] substituted $p_{N}(t)(1.2)$ for $f(t)$ in (1.1) to obtain an approximation $Q_{N}^{C K}(f ; c)$ to $Q(f ; c)$ as follows:

$$
Q_{N}^{C K}(f ; c)=\sum_{k=0}^{N}{ }^{\prime \prime} a_{k}^{N} Q\left(T_{k} ; c\right),
$$

where the modified moment $Q\left(T_{k} ; c\right)=f_{-1}^{1} T_{k}(t) /(t-c) d t$ can be computed by means of a three-term recurrence relation [1]. However, this method is not suitable for our purpose because the error $Q(f ; c)-Q_{N}^{C K}(f ; c)$ cannot be bounded independently of the value of $c$ [3].

On the other hand, subtracting out the singularity [5, p.184], [7, p. 104], [18], [19], one can write $Q(f ; c)(1.1)$ in the form

$$
Q(f ; c)=\int_{-1}^{1} g_{c}(t) d t+f(c) \log \left(\frac{1-c}{1+c}\right),
$$

where $g_{c}(t)$ is defined by

$$
g_{c}(t)=\{f(t)-f(c)\} /(t-c) .
$$

Chawla and Jayarajan [2], and subsequently Kumar [14], made use of the approximate polynomial $p_{N}(t)(1.2)$ to interpolate $g_{c}(t)$ instead of $f(t)$ at $t_{j}^{N}$ and obtained the quadrature formulae

$$
Q_{N}^{C J}(f ; c)=\sum_{j=0}^{N}{ }^{\prime \prime} A_{j}^{N} g_{c}\left(t_{j}^{N}\right)+f(c) \log \left(\frac{1-c}{1+c}\right),
$$

when $t_{j}^{N} \neq c$ for all $j$. In the above, $A_{j}^{N}$ are given by

$$
A_{j}^{N}=\frac{4}{N} \sum_{k=0}^{N / 2} T_{2 k}\left(t_{j}^{N}\right) /\left(1-4 k^{2}\right), \quad 0 \leq j \leq N,
$$

where here and henceforth we conveniently assume that $N$ is even.

It is known [14] that the quadrature formulae (1.7) can yield, in general, better approximate values for (1.1) than the formulae (1.4), but in the computation of $g_{c}\left(t_{j}^{N}\right)$, we have severe numerical cancellation if a node $t_{j}^{N}$ happens to be very close to $c$ [9], [15]. This instability requires special care in programming the function $g_{c}$. 
We now show that we can avoid this instability by approximating $f(t)$ and $f(c)$ in (1.6) by $p_{N}(t)$ and $p_{N}(c)$ (1.2), respectively; the approximation $Q_{N}(f ; c)$ to the integral $Q(f ; c)$ then becomes

$$
Q_{N}(f ; c)=\int_{-1}^{1} \frac{p_{N}(t)-p_{N}(c)}{t-c} d t+f(c) \log \left(\frac{1-c}{1+c}\right) .
$$

Expanding the integrand in (1.8) in Chebyshev polynomials,

$$
\frac{p_{N}(t)-p_{N}(c)}{t-c}=\sum_{k=0}^{N-1} d_{k} T_{k}(t)
$$

and integrating term by term, yields a new integration formula

$$
Q_{N}(f ; c)=2 \sum_{k=0}^{N / 2-1}{ }^{\prime} d_{2 k} /\left(1-4 k^{2}\right)+f(c) \log \left(\frac{1-c}{1+c}\right)
$$

where the prime denotes the summation whose first term is halved. The coefficients $d_{k}$ in (1.9) can be stably computed by using the recurrence relation

$$
d_{k+1}-2 c d_{k}+d_{k-1}=2 a_{k}^{N}, \quad k=N, N-1, \ldots, 1,
$$

in the backward direction with the starting values $d_{N}=d_{N+1}=0$, where we take $a_{N}^{N} / 2$ instead of $a_{N}^{N}$. We have omitted the dependence of $d_{k}$ on $N$ and $c$.

It is well known that the Fast Fourier Transform (FFT) is useful for efficiently computing the coefficients $\left\{a_{k}^{N}\right\}$ in (1.2); see also (2.1) below, [1] and [10], where by doubling $N$ the computation can be repeated, reusing the previous values until an error criterion is satisfied. It is advantageous to have more chances of checking the stopping criterion than by doubling $N$, in order to enhance the efficiency of automatic quadrature. In [12], we allowed $N$ to take the forms $3 \times 2^{n}$ and $5 \times 2^{n}$ as well as $2^{n}$, that is,

$$
N=3,4,5, \ldots, 3 \times 2^{n}, 4 \times 2^{n}, 5 \times 2^{n}, \ldots(n=1,2, \ldots) .
$$

In $\S 2$ we briefly review how to generate recursively the sequence of the interpolating polynomials $\left\{p_{N}(t)\right\}$ by increasing $N$ as in (1.12) and by using the FFT. The set of the $N+1$ nodes $u_{j}^{N}(0 \leq j \leq N)$ for $p_{N}(t)$ is chosen to be a subset of $\left\{\cos \pi j / 2^{m}\right\} \quad\left(0 \leq j \leq 2^{m}\right)$ used in the CC method, where $m$ is the smallest integer such that $N \leq 2^{m}$.

We remark that the present quadrature rule $Q_{N}(f ; c)(1.8)$ or $(1.10)$ is not of interpolatory type because the degree of exactness in the present rule, using $N+2$ abscissae, $u_{j}^{N}(0 \leq j \leq N)$ and $c$, is $N$, not $N+1$. As will be shown in $\S 3$, however, since the function value $f(c)$ is used in the quadrature rule $Q_{N}(f ; c)$ (1.8), but not in interpolating $f(t)$, the error of $Q_{N}(f ; c)$ can be bounded independently of the value of $c$ for smooth functions $f(t)$. See (3.8), (3.10), and (3.11) below. This fact enables us to use the polynomial $p_{N}(t)$ common to the set of the approximations $\left\{Q_{N}(f ; c)\right\}$ for a set of $c$-values 
in $(-1,1)$. In $\S 4$ numerical comparisons with other automatic quadrature methods are shown.

\section{Computation of the Chebyshev coefficients}

We will outline the iterative procedure for computing the sequence $\left\{p_{N}(t)\right\}$ (1.2) of the truncated Chebyshev series by increasing $N$ as in (1.12). For details, see [12].

We begin with the sample points for $p_{N}(t)$ to interpolate $f(t)$. If the sample points are carefully chosen, the interpolating polynomial converges [13, p. 254]. We gave in [11] and [12] a sequence $\left\{\beta_{j}\right\}$ which is a modification of the van der Corput sequence and satisfies the recurrence relation:

$$
\beta_{2 j}=\beta_{j} / 2, \quad \beta_{2 j+1}=\beta_{2 j}+1 / 2, \quad j=1,2, \ldots,
$$

with the starting value $\beta_{1}=3 / 4$. The set of the sample points $\left\{\cos 2 \pi \beta_{j}\right\}$ $(j=-1,0,1, \ldots)$, where we put $\beta_{-1}=0$ and $\beta_{0}=1 / 2$, is a sequence of Chebyshev points [13, p. 254], which makes the sequence of interpolating polynomials converge uniformly on $[-1,1]$ for functions analytic on $[-1,1]$. The polynomial $p_{N}(t)$ is determined so as to interpolate $f(t)$ at the first $N+1$ points of the sequence $\left\{\cos 2 \pi \beta_{j}\right\}(j=-1,0,1, \ldots)$.

Let $N=2^{n}(n=2,3, \ldots)$; then the set of the $N+1$ abscissae $\left\{\cos 2 \pi \beta_{j}\right\}$ $(-1 \leq j<N)$ coincides with the zeros of $\omega_{N+1}(t)(1.3)$, that is, $\{\cos \pi j / N\}$ $(0 \leq j \leq N)$ used in the CC method. Therefore, the interpolation condition

$$
p_{N}(\cos \pi j / N)=f(\cos \pi j / N), \quad 0 \leq j \leq N,
$$

determines the coefficients $a_{k}^{N}$ for $p_{N}(t)(1.2)$ as follows:

$$
a_{k}^{N}=\frac{2}{N} \sum_{j=0}^{N} \prime \prime f(\cos \pi j / N) \cos (\pi k j / N), \quad 0 \leq k \leq N .
$$

It is known that the right-hand side of $(2.1)$ can be efficiently computed by means of the FFT for real data [10].

We represent the polynomials $p_{5 N / 4}(t)$ and $p_{3 N / 2}(t)$ interpolating $f(t)$ at the nodes $\left\{\cos 2 \pi \beta_{j}\right\}$, where $-1 \leq j<N+N / 4$ for $p_{5 N / 4}(t)$ and $-1 \leq j<$ $N+N / 2$ for $p_{3 N / 2}(t)$, respectively, in the form

$$
\begin{aligned}
p_{5 N / 4}(t)-p_{N}(t) & =-\omega_{N+1}(t) \sum_{k=1}^{N / 4} b_{k}^{N} U_{k-1}(t) \\
& =\sum_{k=1}^{N / 4} b_{k}^{N}\left\{T_{N-k}(t)-T_{N+k}(t)\right\},
\end{aligned}
$$




$$
\begin{aligned}
p_{3 N / 2}(t)-p_{N}(t) & =-\omega_{N+1}(t) \sum_{k=1}^{N / 2} B_{k}^{N} U_{k-1}(t) \\
& =\sum_{k=1}^{N / 2} B_{k}^{N}\left\{T_{N-k}(t)-T_{N+k}(t)\right\} .
\end{aligned}
$$

Then, the coefficients $\left\{b_{k}^{N}\right\}$ and $\left\{B_{k}^{N}\right\}$ are determined to satisfy the conditions

$$
\begin{array}{cl}
p_{5 N / 4}\left(v_{j}^{N}\right)=f\left(v_{j}^{N}\right), & 0 \leq j<N / 4, \\
p_{3 N / 2}\left(w_{j}^{N}\right)=f\left(w_{j}^{N}\right), & 0 \leq j<N / 2,
\end{array}
$$

where the sample points $v_{j}^{N}$ and $w_{j}^{N}$ are defined by

$$
\begin{gathered}
v_{j}^{N}=\cos 8 \pi\left(j+\beta_{4}\right) / N \quad \text { or } \quad T_{N / 4}\left(v_{j}^{N}\right)-\cos 2 \pi \beta_{4}=0, \\
w_{j}^{N}=\cos 4 \pi\left(j+\beta_{2}\right) / N \quad \text { or } \quad T_{N / 2}\left(w_{j}^{N}\right)-\cos 2 \pi \beta_{2}=0,
\end{gathered}
$$

respectively. This is because the set of the additional $N / 4(N / 2)$ abscissae $\left\{\cos 2 \pi \beta_{j}\right\}, N \leq j<N / 4(N \leq j<N / 2)$ for $p_{5 N / 4}(t)\left(p_{3 N / 2}(t)\right)$ coincides with $\left\{v_{j}^{N}\right\}, 0 \leq j<N / 4\left(\left\{w_{j}^{N}\right\}, 0 \leq j<N / 2\right)$ [12]. If the set of $N / 2$ sample points $\left\{\cos 4 \pi\left(j+\beta_{3}\right) / N\right\}(0 \leq j<N / 2)$, which agrees with $\left\{\cos 2 \pi \beta_{j}\right\}$ $(3 N / 2 \leq j<2 N)$, is added to the set of abscissae for $p_{3 N / 2}(t)$, we have $2 N+$ 1 abscissae $\{\cos \pi j /(2 N)\}(0 \leq j \leq 2 N)$ for $p_{2 N}(t)$. Thus the sequence of the interpolating polynomials $\left\{p_{3 m}(t), p_{4 m}(t), p_{5 m}(t), \ldots\right\}\left(m=2^{n}, n=\right.$ $1,2, \ldots)$ is recursively generated. The FFT [12] is used to efficiently compute the coefficients $\left\{b_{k}^{N}\right\}$ and $\left\{B_{k}^{N}\right\}$.

\section{ERROR ESTIMATES}

Assume that $N=2^{n}(n=2,3, \ldots)$ and define $A_{k}^{N}$ by

$$
A_{k}^{N}= \begin{cases}a_{k}^{N}, & 0 \leq k<N-N / 4, \\ a_{k}^{N}+b_{N-k}^{N}, & N-N / 4 \leq k<N, \\ a_{N}^{N} / 2, & k=N, \\ -b_{k-N}^{N}, & N<k \leq N+N / 4 .\end{cases}
$$

Then, the approximate quadrature $Q_{5 N / 4}(f ; c)$ depending on the polynomial $p_{5 N / 4}(t)(2.2)$ is given by the right-hand side of $(1.10)$, where the sum ranges from 0 to $N / 2+N / 8-1$, and by (1.11) with $a_{k}^{N}$ replaced by $A_{k}^{N}$ (3.1). Similarly, one can obtain the approximation $Q_{3 N / 2}(f ; c)$ depending on the polynomial $p_{3 N / 2}(t)(2.3)$.

Now, we will give error estimates for the approximations $Q_{N}(f ; c)$, $Q_{5 N / 4}(f ; c)$, and $Q_{3 N / 2}(f ; c)$, especially for analytic functions $f$. Let 
$\varepsilon_{\rho}$ denote the ellipse in the complex plane $z=x+i y$ with foci $(x, y)=$ $(-1,0),(1,0)$ and semimajor axis $a=\left(\rho+\rho^{-1}\right) / 2$ and semiminor axis $b=$ $\left(\rho-\rho^{-1}\right) / 2$ for a constant $\rho>1$.

Assume that $f(z)$ is single-valued and analytic inside and on $\varepsilon_{\rho}$. Then, the error of the interpolating polynomial $p_{N}(t)$ can be expressed in terms of a contour integral [6], [7, p. 105], [8], which is also expanded in a Chebyshev series [11]:

$$
f(t)-p_{N}(t)=\frac{1}{2 \pi i} \oint_{\varepsilon_{\rho}} \frac{\omega_{N+1}(t) f(z) d z}{(z-t) \omega_{N+1}(z)}=\omega_{N+1}(t) \sum_{k=0}^{\infty}{ }^{\prime} V_{k}^{N}(f) T_{k}(t),
$$

where the coefficients $V_{k}^{N}(f)$ are given by

$$
V_{k}^{N}(f)=\frac{1}{\pi^{2} i} \oint_{\varepsilon_{\rho}} \frac{\tilde{U}_{k}(z) f(z) d z}{\omega_{N+1}(z)}, \quad k \geq 0 .
$$

The Chebyshev function of the second kind, $\widetilde{U}_{k}(z)$, is defined by

$$
\tilde{U}_{k}(z)=\int_{-1}^{1} \frac{T_{k}(t) d t}{(z-t) \sqrt{1-t^{2}}}=\frac{\pi}{\sqrt{z^{2}-1} w^{k}}=\frac{2 \pi}{\left(w-w^{-1}\right) w^{k}},
$$

where $w=z+\sqrt{z^{2}-1}$ and $|w|>1$ for $z \notin[-1,1]$ [8], [11].

Using (3.2) in (1.5), (1.6) and (1.8) yields the error for the approximate integral $Q_{N}(f ; c)$ :

$$
Q(f ; c)-Q_{N}(f ; c)=\sum_{k=0}^{\infty} \Omega_{k}^{N}(c) V_{k}^{N}(f)
$$

where $\Omega_{k}^{N}(c)$ is given by

$$
\Omega_{k}^{N}(c)=\int_{-1}^{1} \frac{\omega_{N+1}(t) T_{k}(t)-\omega_{N+1}(c) T_{k}(c)}{t-c} d t, \quad k \geq 0 .
$$

In Appendix A we prove the following lemma.

Lemma 3.1. Let $N=2^{n}, n=2,3, \ldots$, and $\Omega_{k}^{N}(c)$ be defined by (3.6). Then, $\Omega_{k}^{N}(c)$ is bounded independently of the value of $c$ as well as $N$ and $k$; indeed,

$$
\left|\Omega_{k}^{N}(c)\right| \leq 8 .
$$

From (3.5) and (3.7) we have the following theorem.

Theorem 3.2. Let $N=2^{n}, n=2,3, \ldots$, and assume that $f(z)$ is singlevalued and analytic inside and on $\varepsilon_{\rho}$. Then, the error of the approximate integral $Q_{N}(f ; c)$ given by $(1.10)$ is bounded independently of $c$ by

$$
\left|Q(f ; c)-Q_{N}(f ; c)\right| \leq 8 \sum_{k=0}^{\infty}\left|V_{k}^{N}(f)\right|,
$$

where $V_{k}^{N}(f)$ is given by (3.3). 
Similarly, the errors of the approximate integrals $Q_{5 N / 4}(f ; c)$ and $Q_{3 N / 2}(f ; c)$ are bounded as follows:

Theorem 3.3. Let $N=2^{n} \quad(n=2,3, \ldots)$ and assume that $f(z)$ is singlevalued and analytic inside and on $\varepsilon_{\rho}$. Further, let $V_{k}^{N+N / \sigma}(f)(\sigma=2,4)$ be defined by

$$
\begin{array}{r}
V_{k}^{N+N / \sigma}(f)=\frac{1}{\pi^{2} i} \oint_{\varepsilon_{\rho}} \frac{\tilde{U}_{k}(z) f(z) d z}{\omega_{N+1}(z)\left\{T_{N / \sigma}(z)-\cos 2 \pi \beta_{\sigma}\right\}}, \\
k \geq 0, \quad \sigma=2,4 .
\end{array}
$$

Then, we have

$$
\begin{aligned}
\left|Q(f ; c)-Q_{5 N / 4}(f ; c)\right| & \leq 8\left(1+\left|\cos 2 \pi \beta_{4}\right|\right) \sum_{k=0}^{\infty}{ }^{\prime}\left|V_{k}^{N+N / 4}(f)\right| \\
& \sim 11.1 \sum_{k=0}^{\infty}{ }^{\prime}\left|V_{k}^{N+N / 4}(f)\right|, \\
\left|Q(f ; c)-Q_{3 N / 2}(f ; c)\right| & \leq 8\left(1+\left|\cos 2 \pi \beta_{2}\right|\right) \sum_{k=0}^{\infty}\left|V_{k}^{N+N / 2}(f)\right| \\
& \sim 13.7 \sum_{k=0}^{\infty}{ }^{\prime}\left|V_{k}^{N+N / 2}(f)\right|,
\end{aligned}
$$

where $\beta_{4}=3 / 16$ and $\beta_{2}=3 / 8$.

Proof. The error of the interpolating polynomial $p_{N+N / \sigma}(t)(\sigma=2,4)$ has an expression similar to $(3.2)$ :

$$
\begin{aligned}
f(t)-p_{N+N / \sigma}(t)= & \frac{1}{2 \pi i} \oint_{\varepsilon_{\rho}} \frac{\omega_{N+1}(t)\left\{T_{N / \sigma}(t)-\cos 2 \pi \beta_{\sigma}\right\} f(z) d z}{(z-t) \omega_{N+1}(z)\left\{T_{N / \sigma}(z)-\cos 2 \pi \beta_{\sigma}\right\}} \\
= & \omega_{N+1}(t)\left\{T_{N / \sigma}(t)-\cos 2 \pi \beta_{\sigma}\right\} \\
& \times \sum_{k=0}^{\infty}{ }_{k}^{N+N / \sigma}(f) T_{k}(t), \quad \sigma=2,4,
\end{aligned}
$$

where $V_{k}^{N+N / \sigma}(f)$ is given by (3.9). If we note in (3.12) that

$$
\begin{aligned}
& 2 \omega_{N+1}(t)\left\{T_{N / \sigma}(t)-\cos 2 \pi \beta_{\sigma}\right\} \\
& \quad=\omega_{N+N / \sigma+1}(t)+\omega_{N-N / \sigma+1}(t)-2 \cos 2 \pi \beta_{\sigma} \omega_{N+1}(t),
\end{aligned}
$$

then the proof of (3.10) and (3.11) is established in a way similar to that for (3.8).

Suppose that $f(z)$ is a meromorphic function which has $M$ simple poles at the points $z_{m}(m=1,2, \ldots, M)$ outside of $\varepsilon_{\rho}$ with residues $\operatorname{Res} f\left(z_{m}\right)$. 
Then, performing the contour integral of (3.3) gives

$$
V_{k}^{N}(f)=-\frac{2}{\pi} \sum_{m=1}^{M} \operatorname{Res} f\left(z_{m}\right) \tilde{U}_{k}\left(z_{m}\right) / \omega_{N+1}\left(z_{m}\right), \quad k \geq 0 .
$$

Put $z=\left(w+w^{-1}\right) / 2$; then the Chebyshev polynomial can be expressed as

$$
T_{n}(z)=\left(w^{n}+w^{-n}\right) / 2, \quad w=z+\sqrt{z^{2}-1}, \quad|w|>1 \text { for } z \notin[-1,1] .
$$

From (1.3), (3.4), (3.14) and (3.15) it is seen that $\left|V_{k}^{N}(f)\right|=O\left(r^{-k-N}\right)$, where $r=\min _{1 \leq m \leq M}\left|z_{m}+\sqrt{z_{m}^{2}-1}\right|>1$. Thus, from (3.8) we may estimate the error for $Q_{N}(f ; c)$ as follows:

$$
\left|Q(f ; c)-Q_{N}(f ; c)\right| \lesssim 4\left|V_{0}^{N}(f)\right|(r+1) /(r-1) .
$$

Now, we wish to estimate $\left|V_{0}^{N}(f)\right|$ in terms of the available coefficients $a_{k}^{N}$ of the truncated Chebyshev series $p_{N}(t)$ (1.2). Elliott [6] gives the expression

$$
a_{k}^{N}=\frac{2}{\pi i} \oint_{\varepsilon_{\rho}} \frac{T_{N-k}(z) f(z)}{\omega_{N+1}(z)} d z, \quad 0 \leq k \leq N .
$$

Performing the contour integral in (3.17) and comparing with (3.14) gives the estimates

$$
\left|V_{0}^{N}\right| \sim\left|a_{N}^{N}\right| r /\left(r^{2}-1\right)
$$

and $\left|a_{k}^{N}\right| \sim r\left|a_{k+1}^{N}\right|$, unless the poles $z_{m}$ of $f(z)$ are close to the segment $[-1,1]$ on the real axis. Finally, from (3.16) and (3.18) we could obtain an estimate of the truncation error $E_{N}(f ; c)$ for $Q_{N}(f ; c)$ as follows:

$$
E_{N}(f ; c)=8\left(\left|a_{N}^{N}\right| / 2\right) r /(r-1)^{2},
$$

where we note that $a_{N}^{N} / 2$ is the coefficient of the last term in the truncated Chebyshev series (1.2). The constant $r$ may be estimated from the asymptotic behavior of $\left\{a_{k}^{N}\right\}$ in a way similar to that in the stopping criterion described in [12].

If $\left|a_{k}^{N}\right|$ decreases slowly as $k$ increases, that is, $r \rightarrow 1+$, we prefer a rather cautious error estimation similar to that given in the stopping criterion of [12] in place of (3.19). See also [16].

Next, we turn to estimate the error (3.10) of $Q_{5 N / 4}(f ; c)$ in terms of the available coefficients $b_{k}^{N}$ of $p_{5, N / 4}(t)(2.2)$.

Lemma 3.4. Let $f(z)$ be single-valued and analytic inside and on $\varepsilon_{\rho}$. Further, define

$$
\begin{aligned}
J_{k}^{N}(\sigma)=\frac{-1}{\pi i} \oint_{\varepsilon_{\rho}} \frac{T_{N / \sigma-k}(z) f(z) d z}{\omega_{N+1}(z)\left\{T_{N / \sigma}(z)-\cos 2 \pi \beta_{\sigma}\right\}}, \\
1 \leq k \leq N / \sigma, \sigma=2,4,
\end{aligned}
$$


where the right-hand side of (3.20) is multiplied by $1 / 2$ when $k=N / \sigma$. Then, for $b_{k}^{N}$ in (2.2) and $B_{k}^{N}$ in (2.3), we have $b_{k}^{N}=J_{k}^{N}(4)$ and $B_{k}^{N}=J_{k}^{N}(2)$, respectively.

Proof. From (3.2) and (3.12) we have

$$
\begin{aligned}
& p_{N+N / \sigma}(t)-p_{N}(t)=\frac{1}{2 \pi i} \oint_{\varepsilon_{\rho}} \frac{\omega_{N+1}(t)\left\{T_{N / \sigma}(z)-T_{N / \sigma}(t)\right\} f(z) d z}{(z-t) \omega_{N+1}(z)\left\{T_{N / \sigma}(z)-\cos 2 \pi \beta_{\sigma}\right\}} \\
&=\frac{1}{\pi i} \sum_{n=0}^{N / \sigma-1} \oint_{\varepsilon_{\rho}} \frac{\omega_{N+1}(t) U_{N / \sigma-n-1}(t) T_{n}(z) f(z) d z}{\omega_{N+1}(z)\left\{T_{N / \sigma}(z)-\cos 2 \pi \beta_{\sigma}\right\}} \\
& \sigma=2,4
\end{aligned}
$$

In deriving the second equality above we have used the identity (A.3) in Appendix $\mathrm{A}$, where we take $N / \sigma$, a complex $z$ and real $t$ for $k+1, t$, and $c$, respectively. Comparing (2.2), (2.3) and (3.21) establishes Lemma 3.4.

Performing the contour integrals in (3.9) and (3.20) and comparing both results yields the estimates

$$
\left|V_{0}^{N+N / 4}\right| \sim 4\left|b_{N / 4}^{N}\right| r /\left(r^{2}-1\right)
$$

$\left|V_{k}^{N+N / 4}(f)\right|=O\left(r^{-k-N-N / 4}\right)$ and $\left|b_{k}^{N}\right| \sim r\left|b_{k+1}^{N}\right|$. Using these relations in (3.10), one gets an estimate of the truncation error $E_{N+N / 4}(f ; c)$ for $Q_{5 N / 4}(f ; c)$ as follows:

$$
E_{5 N / 4}(f ; c)=22.2\left|b_{N / 4}^{N}\right| r /(r-1)^{2}
$$

Similarly, it follows that

$$
E_{3 N / 2}(f ; c)=27.4\left|B_{N / 2}^{N}\right| r /(r-1)^{2}
$$

If the constant $r$ is found to be close or equal to 1 , we resort to a check procedure; see the stopping criterion in [12].

It should be noted that the error estimates (3.19), (3.23) and (3.24) for the quadrature rules $Q_{N}(f ; c), Q_{5 N / 4}(f ; c)$, and $Q_{3 N / 2}(f ; c)$, respectively, are independent of the value of $c$. This fact enables us to use the approximate polynomial $p_{N}(t), p_{5 N / 4}(t)$ or $p_{3 N / 2}(t)$ common to the set of the integrals $Q(f ; c)(1.1)$ for a set of $c$-values if a stopping criterion is satisfied. 


\section{Numerical EXAMPLES}

We now show numerical results obtained with the present automatic quadrature scheme for the following test problems:

$$
\begin{array}{ll}
f_{-1}^{1} \frac{\exp \{a(t-1)\}}{t-c} d t, & a=4,8,16, \\
f_{-1}^{1} \frac{\left(t^{2}+a^{2}\right)^{-1}}{t-c} d t, & a=1,1 / 4,1 / 8, \\
f_{0}^{1} \frac{\cos 2 \pi a t}{t-c} d t, & a=8,16,32, \\
f_{-1}^{1} \frac{1-a^{2}}{1-2 a t+a^{2}} \cdot \frac{1}{t-c} d t, & a=0.8,0.9,0.95, \\
f_{0}^{1} \frac{\sqrt{1-t^{2}}}{t-c} d t . &
\end{array}
$$

TABLE 1

Comparison of the performance of the present method with $Q A W C$ in QUADPACK [17] for $f_{-1}^{1} e^{a(t-1)} /(t-c) d t, a=4,8,16 . N$ denotes the number of abscissae required to satisfy the tolerance $\varepsilon_{a}$. The present method computes all the integrals for a set of the values of $c$ by using $N-1$ abscissae once and for all, and by using the number of the corresponding values of $c$.

\begin{tabular}{|c|c||c|c|c|c|c|c|c|c|}
\hline \multicolumn{9}{|c||}{} & \multicolumn{5}{c|}{$\varepsilon_{a}=10^{-6}$} & \multicolumn{4}{c|}{$\varepsilon_{a}=10^{-10}$} \\
\hline \multicolumn{2}{|c||}{} & present method & \multicolumn{2}{c|}{ QUADPACK } & present method & QUADPACK \\
\hline$a$ & $c$ & $N$ & error & $N$ & error & $N$ & error & $N$ & error \\
\hline \multirow{4}{*}{4} & 0.2 & $\Uparrow^{(+1)}$ & $1 \times 10^{-10}$ & 25 & $2 \times 10^{-15}$ & $\Uparrow^{(+1)}$ & $9 \times 10^{-15}$ & 105 & $3 \times 10^{-13}$ \\
& 0.5 & $17+1$ & $2 \times 10^{-11}$ & 25 & $4 \times 10^{-15}$ & $21+1$ & $1 \times 10^{-16}$ & 105 & $4 \times 10^{-14}$ \\
& 0.95 & $\Downarrow(+1)$ & $6 \times 10^{-11}$ & 25 & $1 \times 10^{-14}$ & $\Downarrow(+1)$ & $2 \times 10^{-14}$ & 105 & $2 \times 10^{-13}$ \\
\hline \multirow{4}{*}{8} & 0.2 & $\Uparrow^{(+1)}$ & $5 \times 10^{-10}$ & 105 & $7 \times 10^{-13}$ & $\uparrow^{(+1)}$ & $9 \times 10^{-13}$ & 145 & $7 \times 10^{-13}$ \\
& 0.5 & $21+1$ & $3 \times 10^{-10}$ & 105 & $2 \times 10^{-15}$ & $25+1$ & $3 \times 10^{-13}$ & 185 & $4 \times 10^{-13}$ \\
& 0.95 & $\Downarrow(+1)$ & $8 \times 10^{-11}$ & 65 & $4 \times 10^{-15}$ & $\Downarrow(+1)$ & $1 \times 10^{-12}$ & 145 & $2 \times 10^{-13}$ \\
\hline \multirow{4}{*}{16} & 0.2 & $\Uparrow^{(+1)}$ & $7 \times 10^{-13}$ & 105 & $7 \times 10^{-13}$ & $\Uparrow^{(+1)}$ & $7 \times 10^{-13}$ & 185 & $7 \times 10^{-13}$ \\
& 0.5 & $33+1$ & $6 \times 10^{-13}$ & 145 & $8 \times 10^{-13}$ & $33+1$ & $6 \times 10^{-13}$ & 225 & $8 \times 10^{-13}$ \\
& 0.95 & $\Downarrow(+1)$ & $2 \times 10^{-14}$ & 105 & $6 \times 10^{-16}$ & $\Downarrow(+1)$ & $2 \times 10^{-14}$ & 185 & $1 \times 10^{-13}$ \\
\hline
\end{tabular}




\section{TABLE 2}

Comparison of the performance of the present method with $Q A W C$ in QUADPACK [17] for $f_{-1}^{1}\left(t^{2}+a^{2}\right)^{-1} /(t-c) d t, a=1,1 / 4,1 / 8$.

\begin{tabular}{|c|c||c|c|c|c|c|c|c|c|}
\hline \multicolumn{2}{|c||}{} & \multicolumn{5}{c|}{$\varepsilon_{a}=10^{-6}$} & \multicolumn{4}{c|}{$\varepsilon_{a}=10^{-10}$} \\
\hline \multicolumn{2}{|c||}{} & present method & \multicolumn{2}{c|}{ QUADPACK } & present method & \multicolumn{2}{c|}{ QUADPACK } \\
\hline$a$ & $c$ & $N$ & error & $N$ & error & $N$ & error & $N$ & error \\
\hline \multirow{4}{*}{1} & 0.2 & $\Uparrow^{(+1)}$ & $1 \times 10^{-8}$ & 65 & $4 \times 10^{-13}$ & $\Uparrow^{(+1)}$ & $6 \times 10^{-13}$ & 145 & $4 \times 10^{-13}$ \\
& 0.5 & $\mathbf{2 1 + 1}$ & $1 \times 10^{-8}$ & 65 & $5 \times 10^{-13}$ & $\mathbf{3 3 + 1}$ & $5 \times 10^{-13}$ & 145 & $5 \times 10^{-13}$ \\
& 0.95 & $\Downarrow(+1)$ & $4 \times 10^{-9}$ & 65 & $5 \times 10^{-13}$ & $\Downarrow(+1)$ & $1 \times 10^{-13}$ & 105 & $7 \times 10^{-13}$ \\
\hline \multirow{4}{*}{$1 / 4$} & 0.2 & $\Uparrow^{(+1)}$ & $3 \times 10^{-7}$ & 225 & $2 \times 10^{-11}$ & $\Uparrow^{(+1)}$ & $7 \times 10^{-13}$ & 365 & $3 \times 10^{-12}$ \\
& 0.5 & $\mathbf{8 1 + 1}$ & $3 \times 10^{-8}$ & 215 & $9 \times 10^{-12}$ & $\mathbf{1 2 9 + 1}$ & $5 \times 10^{-13}$ & 325 & $3 \times 10^{-12}$ \\
& 0.95 & $\Downarrow(+1)$ & $9 \times 10^{-10}$ & 165 & $1 \times 10^{-11}$ & $\Downarrow(+1)$ & $1 \times 10^{-13}$ & 235 & $2 \times 10^{-12}$ \\
\hline \multirow{4}{*}{$1 / 8$} & 0.2 & $\Uparrow{ }^{(+1)}$ & $4 \times 10^{-7}$ & 335 & $6 \times 10^{-12}$ & $\Uparrow{ }^{(+1)}$ & $1 \times 10^{-12}$ & 505 & $1 \times 10^{-11}$ \\
& 0.5 & $\mathbf{1 6 1 + 1}$ & $3 \times 10^{-7}$ & 225 & $3 \times 10^{-11}$ & $\mathbf{2 5 7 + 1}$ & $2 \times 10^{-13}$ & 445 & $1 \times 10^{-12}$ \\
& 0.95 & $\Downarrow(+1)$ & $1 \times 10^{-7}$ & 255 & $5 \times 10^{-12}$ & $\Downarrow(+1)$ & $5 \times 10^{-13}$ & 325 & $1 \times 10^{-11}$ \\
\hline
\end{tabular}

Tables 1 - 5 compare the results of the present scheme with those of QAWC in the subroutine package QUADPACK [17] for each problem (4.1)-(4.5). We show the number of function evaluations $N$ required to satisfy the requested absolute accuracy $\varepsilon_{a}$ for each integral and the actual errors.

It should be noted that the present scheme can efficiently give all the approximations to the integrals (1.1) for a set of $c$-values by using the common number of function evaluations once and for all, except for each function value $f(c)$ at $c$, for smooth functions $f(t)$. Consequently, in each Table 1-5, the present method requires only $N+$ extra $2(=N+2)$ function evaluations to compute the three integrals for the three values of $c$. For example, in Table 1, $20\{=N+2=(17+1)+2\}$ function evaluations are sufficient for the three integrals with the parameter $a=4$ to satisfy the tolerance $\varepsilon_{a}=10^{-6}$.

The computation was carried out in double-precision arithmetic (about 16 significant digits). 
TABLE 3

Comparison of the performance of the present method with $Q A W C$ in QUADPACK [17] for $f_{0}^{1} \cos 2 \pi a t /(t-c) d t, a=8,16,32$.

\begin{tabular}{|c|c||c|c|c|c|c|c|c|c|}
\hline \multicolumn{9}{|c||}{} & \multicolumn{5}{c|}{$\varepsilon_{a}=10^{-6}$} & \multicolumn{4}{c|}{$\varepsilon_{a}=10^{-10}$} \\
\hline \multicolumn{2}{|c||}{} & present method & \multicolumn{2}{c|}{ QUADPACK } & present method & QUADPACK \\
\hline$a$ & $c$ & $N$ & error & $N$ & error & $N$ & error & $N$ & error \\
\hline \multirow{4}{*}{8} & 0.6 & $\Uparrow^{(+1)}$ & $2 \times 10^{-10}$ & 325 & $2 \times 10^{-11}$ & $\Uparrow^{(+1)}$ & $1 \times 10^{-13}$ & 495 & $4 \times 10^{-12}$ \\
& 0.8 & $49+1$ & $1 \times 10^{-10}$ & 355 & $1 \times 10^{-11}$ & $\mathbf{6 5 + 1}$ & $1 \times 10^{-13}$ & 425 & $5 \times 10^{-12}$ \\
& 0.95 & $\Downarrow(+1)$ & $3 \times 10^{-11}$ & 305 & $3 \times 10^{-12}$ & $\Downarrow(+1)$ & $2 \times 10^{-13}$ & 505 & $4 \times 10^{-12}$ \\
\hline \multirow{4}{*}{16} & 0.6 & $\Uparrow^{(+1)}$ & $8 \times 10^{-11}$ & 555 & $2 \times 10^{-11}$ & $\Uparrow^{(+1)}$ & $6 \times 10^{-13}$ & 875 & $2 \times 10^{-13}$ \\
& 0.8 & $\mathbf{8 1 + 1}$ & $1 \times 10^{-10}$ & 635 & $1 \times 10^{-11}$ & $\mathbf{9 7 + 1}$ & $5 \times 10^{-14}$ & 785 & $1 \times 10^{-11}$ \\
& 0.95 & $\Downarrow(+1)$ & $5 \times 10^{-12}$ & 595 & $2 \times 10^{-11}$ & $\Downarrow(+1)$ & $2 \times 10^{-14}$ & 975 & $2 \times 10^{-12}$ \\
\hline \multirow{4}{*}{32} & 0.6 & $\Uparrow^{(+1)}$ & $2 \times 10^{-14}$ & 1055 & $4 \times 10^{-12}$ & $\Uparrow^{(+1)}$ & $2 \times 10^{-14}$ & 1615 & $3 \times 10^{-12}$ \\
& 0.8 & $\mathbf{1 6 1 + 1}$ & $9 \times 10^{-14}$ & 1205 & $5 \times 10^{-12}$ & $161+1$ & $9 \times 10^{-14}$ & 1405 & $7 \times 10^{-12}$ \\
& 0.95 & $\Downarrow(+1)$ & $7 \times 10^{-14}$ & 1125 & $3 \times 10^{-11}$ & $\Downarrow(+1)$ & $7 \times 10^{-14}$ & 1595 & $8 \times 10^{-13}$ \\
\hline
\end{tabular}

TABLE 4

Comparison of the performance of the present method with $Q A W C$ in QUADPACK [17] for $f_{-1}^{1}\left(1-a^{2}\right)\left(1-2 a t+a^{2}\right)^{-1} /(t-c) d t$, $a=0.8,0.9,0.95$.

\begin{tabular}{|c|c||c|c|c|c|c|c|c|c|}
\hline \multicolumn{2}{|c||}{} & \multicolumn{5}{c|}{$\varepsilon_{a}=10^{-6}$} & \multicolumn{4}{c|}{$\varepsilon_{a}=10^{-10}$} \\
\hline \multicolumn{2}{|c||}{} & present method & QUADPACK & present method & QUADPACK \\
\hline \multirow{4}{*}{0.8} & $c$ & $N$ & error & $N$ & error & $N$ & error & $N$ & error \\
\hline & 0.15 & $\Uparrow^{(+1)}$ & $4 \times 10^{-9}$ & 195 & $7 \times 10^{-13}$ & $\Uparrow^{(+1)}$ & $3 \times 10^{-12}$ & 305 & $3 \times 10^{-13}$ \\
& 0.45 & $97+1$ & $4 \times 10^{-9}$ & 205 & $1 \times 10^{-12}$ & $129+1$ & $4 \times 10^{-12}$ & 305 & $4 \times 10^{-13}$ \\
& 0.95 & $\Downarrow(+1)$ & $4 \times 10^{-8}$ & 305 & $2 \times 10^{-12}$ & $\Downarrow(+1)$ & $1 \times 10^{-11}$ & 385 & $2 \times 10^{-12}$ \\
\hline \multirow{4}{*}{0.9} & 0.15 & $\Uparrow^{(+1)}$ & $4 \times 10^{-8}$ & 255 & $5 \times 10^{-13}$ & $\Uparrow^{(+1)}$ & $1 \times 10^{-11}$ & 365 & $3 \times 10^{-13}$ \\
& 0.45 & $193+1$ & $2 \times 10^{-8}$ & 265 & $9 \times 10^{-13}$ & $257+1$ & $2 \times 10^{-11}$ & 375 & $4 \times 10^{-13}$ \\
& 0.95 & $\Downarrow(+1)$ & $9 \times 10^{-8}$ & 335 & $2 \times 10^{-12}$ & $\Downarrow(+1)$ & $1 \times 10^{-10}$ & 445 & $5 \times 10^{-13}$ \\
\hline \multirow{4}{*}{0.95} & 0.15 & $\Uparrow^{(+1)}$ & $6 \times 10^{-9}$ & 315 & $3 \times 10^{-13}$ & $\Uparrow^{(+1)}$ & $6 \times 10^{-15}$ & 425 & $2 \times 10^{-13}$ \\
& 0.45 & $385+1$ & $1 \times 10^{-7}$ & 325 & $6 \times 10^{-13}$ & $641+1$ & $2 \times 10^{-13}$ & 435 & $3 \times 10^{-13}$ \\
& 0.95 & $\Downarrow(+1)$ & $5 \times 10^{-7}$ & 395 & $2 \times 10^{-12}$ & $\Downarrow(+1)$ & $8 \times 10^{-13}$ & 505 & $6 \times 10^{-13}$ \\
\hline
\end{tabular}


TABLE 5

Comparison of the performance of the present method with $Q A W C$ in QUADPACK [17] for $f_{0}^{1} \sqrt{1-t^{2}} /(t-c) d t$. The number in the parentheses indicates failure to achieve the required accuracy.

\begin{tabular}{|c||c|c|c|c|c|c|c|c|}
\hline \multicolumn{1}{|c||}{} & \multicolumn{4}{c|}{$\varepsilon_{a}=10^{-3}$} & \multicolumn{4}{c|}{$\varepsilon_{a}=10^{-5}$} \\
\hline & \multicolumn{1}{|c||}{ present method } & \multicolumn{2}{c|}{ QUADPACK } & \multicolumn{2}{c|}{ present method } & \multicolumn{2}{c|}{ QUADPACK } \\
\hline$c$ & $N$ & error & $N$ & error & $N$ & error & $N$ & error \\
\hline 0.6 & $\Uparrow^{(+1)}$ & $4 \times 10^{-4}$ & $(65)$ & $2 \times 10^{-3}$ & $\Uparrow^{(+1)}$ & $6 \times 10^{-7}$ & 315 & $3 \times 10^{-9}$ \\
0.9 & $97+1$ & $2 \times 10^{-4}$ & 285 & $3 \times 10^{-7}$ & $\mathbf{1 0 2 5 + 1}$ & $6 \times 10^{-6}$ & 405 & $4 \times 10^{-9}$ \\
0.95 & $\Downarrow(+1)$ & $1 \times 10^{-4}$ & 295 & $5 \times 10^{-7}$ & $\Downarrow(+1)$ & $7 \times 10^{-6}$ & 445 & $3 \times 10^{-9}$ \\
\hline
\end{tabular}

APPENDIX A

Here, we prove (3.7). By using the relation

$$
2 T_{n}(t) T_{m}(t)=T_{n+m}(t)+T_{|n-m|}(t), \quad n, m \geq 0,
$$

and the definition of $\omega_{N+1}(t)(1.3)$ in (3.6), it follows that

$$
\begin{aligned}
2 \Omega_{k}^{N}(c)= & \int_{-1}^{1} \frac{\omega_{N+k+1}(t)-\omega_{N+k+1}(c)}{t-c} d t \\
& \pm \int_{-1}^{1} \frac{\omega_{|N-k|+1}(t)-\omega_{|N-k|+1}(c)}{t-c} d t, \quad k \geq 0 .
\end{aligned}
$$

In the above, the plus sign is taken if $N-k \geq 1$ and the minus sign if $k-N \geq 1$. Further, the second term in the right-hand side should be ignored when $k=N$.

Elliott [6] gives the identity involving the Chebyshev polynomial of the second kind $U_{k}(t)$ :

$$
T_{k+1}(t)-T_{k+1}(c)=2(t-c) \sum_{n=0}^{k} U_{k-n}(c) T_{n}(t), \quad k \geq 0 .
$$

Using the identities $U_{k}(t)-U_{k-2}(t)=2 T_{k}(t)(k \geq 1)$, where we define $U_{-1}(t)=0$, and (A.3) in (A.2) gives

$$
\begin{aligned}
\Omega_{k}^{N}(c)= & 2 \sum_{n=0}^{N+k} T_{N+k-n}(c) \int_{-1}^{1} T_{n}(t) d t \\
& \pm 2 \sum_{n=0}^{|N-k|} " T_{|N-k|-n}(c) \int_{-1}^{1} T_{n}(t) d t
\end{aligned}
$$

Thus, $\Omega_{k}^{N}(c)$ is bounded by

$$
\left|\Omega_{k}^{N}(c)\right| \leq 2 \sum_{n=0}^{N+k}\left|\int_{-1}^{1} T_{n}(t) d t\right|+2 \sum_{n=0}^{|N-k|} \prime\left|\int_{-1}^{1} T_{n}(t) d t\right| .
$$

If one notes in (A.5) that the integral $\int_{-1}^{1} T_{n}(t) d t$ equals $2 /\left(1-n^{2}\right)$ if $n$ is even, and vanishes otherwise, it is easy to verify (3.7). 


\section{ACKNOWLEDGMENT}

We are indebted to H. Sugiura for helpful comments.

\section{BIBLIOGRAPHY}

1. M. Branders and R. Piessens, An extension of Clenshaw-Curtis quadrature, J. Comput. Appl. Math. 1 (1975), 55-65.

2. M. M. Chawla and N. Jayarajan, Quadrature formulas for Cauchy principal value integrals, Computing 15 (1975), 347-355.

3. M. M. Chawla and S. Kumar, Convergence of quadratures for Cauchy principal value integrals, Computing 23 (1979), 67-72.

4. C. W. Clenshaw and A. R. Curtis, A method for numerical integration on an automatic computer, Numer. Math. 2 (1960), 197-205.

5. P. J. Davis and P. Rabinowitz, Methods of numerical integration, 2nd ed, Academic Press, Orlando, 1984.

6. D. Elliott, Truncation errors in two Chebyshev series approximations, Math. Comp. 19 (1965), 234-248.

7. W. Gautschi, A survey of Gauss-Christoffel quadrature formulae, E.B. Christoffel, The Influence of his Work on Mathematics and the Physical Sciences (P. Butzer and F. Fehér, eds.), Birkhäuser, Basel, 1981, pp. 72-147.

8. W. Gautschi and R. S. Varga, Error bounds for Gaussian quadrature of analytic functions, SIAM J. Numer. Anal. 20 (1983), 1170-1186.

9. W. Gautschi, M. A. Kovačević, and G. V. Milovanović, The numerical evaluation of singular integrals with coth-kernel, BIT 27 (1987), 389-402.

10. W. M. Gentleman, Implementing Clenshaw-Curtis quadrature II: Computing the cosine transformation, Comm. ACM 15 (1972), 343-346.

11. T. Hasegawa, T. Torii, and I. Ninomiya, Generalized Chebyshev interpolation and its application to automatic quadrature, Math. Comp. 41 (1983), 537-553.

12. T. Hasegawa, T. Torii, and H. Sugiura, An algorithm based on the FFT for a generalized Chebyshev interpolation, Math. Comp. 54 (1990), 195-210.

13. V. I. Krylov, Approximate calculation of integrals, translated by A. H. Stroud, Macmillan, New York, 1962.

14. S. Kumar, A note on quadrature formulae for Cauchy principal value integrals, J. Inst. Math. Appl. 26 (1980), 447-451.

15. G. Monegato, The numerical evaluation of one-dimensional Cauchy principal value integrals, Computing 29 (1982), 337-354.

16. J. Oliver, Doubly-adaptive Clenshaw-Curtis quadrature method, Comput J. 15 (1972), 141-147.

17. R. Piessens, E. deDoncker-Kapenga, C. W. Überhuber, and D. K. Kahaner, QUADPACK, A subroutine package for automatic integration, Springer-Verlag, Berlin, 1983.

18. P. Rabinowitz, Numerical integration in the presence of an interior singularity, J. Comput. Appl. Math. 17 (1987), 31-41.

19. P. Rabinowitz, Convergence results for piecewise linear quadratures for Cauchy principal value integrals, Math. Comp. 51 (1988), 741-747.

Department of Information Science, Faculty of ENGineering, FukUi University, FukUi, 910, JAPAN

E-mail address: hasegawa@fuis.fuis.fukui-u.ac.jp

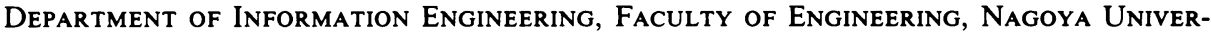
SITY, NAGOYA, 464-01, JAPAN 